\title{
MEASUREMENT AND MODELING OF SOLITARY WAVE INDUCED BED SHEAR STRESS OVER A ROUGH BED
}

\begin{abstract}
Jaya Kumar Seelam ${ }^{1}$ and Tom. E. Baldock ${ }^{2}$
Bed shear stresses generated by solitary waves were measured using a shear cell apparatus over a rough bed in laminar and transitional flow regimes $(\sim 600<\mathrm{Re}<\sim 60200)$. Modeling of bed shear stress was carried out using analytical models employing convolution integration methods forced with the free stream velocity and three eddy viscosity models. The measured wave height to water depth $(\mathrm{h} / \mathrm{d})$ ratio varied between 0.13 and 0.65 ; maximum nearbed velocity varied between 0.16 and $0.47 \mathrm{~m} / \mathrm{s}$ and the maximum total shear stress (sum of form drag and bed shear) varied between 0.565 and $3.29 \mathrm{~Pa}$. Wave friction factors estimated from the bed shear stresses at the maximum bed shear stress using both maximum and instantaneous velocities showed that there is an increase in friction factors estimated using instantaneous velocities, for non-breaking waves. Maximum positive total stress was approximately 2.2 times larger than maximum negative total stress for non-breaking waves. Modeled and measured positive total stresses are well correlated using the convolution model with an eddy viscosity model analogous to steady flow conditions $\left(v_{t}=0.45 u_{*} z_{1}\right.$; where $v_{\mathrm{t}}$ is eddy viscosity, $u_{*}$ is shear velocity and $z_{1}$ is the elevation parameter related to relative roughness). The bed shear stress leads the free stream fluid velocity by approximately $30^{\circ}$ for non-breaking waves and by $48^{\circ}$ for breaking waves, which is under-predicted by $27 \%$ by the convolution model with above mentioned eddy viscosity model.
\end{abstract}

Keywords: bed shear stress; rough bed; bed roughness; shear plate; friction factors; solitary wave

\section{INTRODUCTION}

Solitary wave induced bed shear stresses on a rough bed were measured using a shear plate apparatus. An earlier work by the authors (Seelam et al., 2011) provides a brief review on the importance of solitary wave induced shear stresses, wherein the study was restricted to smooth bed conditions. This earlier study verified the applicability of shear plate apparatus for solitary wave induced shear stresses on smooth bed through a validation using analytical model of Liu et al. (2007) with three eddy viscosity formulations. Very limited studies are available on solitary wave induced bed shear stresses on roughened beds, notable ones being carried out few decades back (Ippen et al., 1955; Naheer, 1978). Ippen et al. (1955) used shear plate and measured the forces on the plate using a force balance and estimated the bed shear stress for smooth as well as rough beds. Naheer (1978) derived mean resistance coefficients for solitary wave flows from energy dissipation considerations. Keulegan (1948) provided a theoretical study on the viscous damping of solitary waves furthered by the work of Liu et al. (2007). Some of the recent studies on solitary wave induced bed shear stress include Barnes et al. (2009) who used a shear plate to measure the total shear force due to solitary bores on sloping bed. Shimozono et al. (2010) used LDV to measure velocity profiles as well as depth-integrated momentum balance to estimate bed shear stress due to solitary waves on a sloped rough bed. In this study, shear stress measurements are directly measured on a fixed horizontal bed and moreover the bed remains always under water unlike the measurements of Barnes et al. (2009) or Shimozono et al. (2010). The advantage of the method adopted in this study over some of the other studies is that a direct measure of the shear stress is obtained in this study without any assumptions of the current profile.

The bed shear stress under solitary wave is derived from direct measurements of shear plate displacement and free stream velocity along with pressure gradients estimated from surface elevation measurements. Measurements were carried out over a fixed bed affixed with sand paper over the measurement section in a wave flume. The derived bed shear stresses were compared with an analytical model as in Liu et al. (2007) and Guard et al. (2010). One of the objectives of this paper is to present new experimental data on solitary wave induced bed shear stress on rough beds measured using shear plate apparatus, and to verify the general applicability of analytical model for the bed shear stress on rough beds. This paper contains the following sections: Methodology adopted for experiments; techniques used for bed shear stress determination; the bed shear stress analytical model and eddy viscosity models used; experimental results and comparison with model data and conclusions.

\footnotetext{
${ }^{1}$ Ocean Engineering, CSIR-National Institute of Oceanography, Dona Paula, Goa, 403004, India

${ }^{2}$ School of Civil Engineering, The University of Queensland, QLD 4072, Australia
} 


\section{METHODOLOGY}

\section{Experimental setup}

Experiments were carried out in a wave flume $(0.75 \mathrm{~m}$ deep; $0.85 \mathrm{~m}$ wide; $20 \mathrm{~m}$ long) at the UQ Gordon McKay hydraulics lab. The setup consisted of a horizontal bed of $11 \mathrm{~m}$ length from the piston type wave paddle followed by a 1:10 slope for $1.6 \mathrm{~m}$ and further horizontal bed for more than $5 \mathrm{~m}$ long before wave absorber (Fig. 1). This flume setup was used to represent a region seaward and landward of the continental slope. The computer controlled wave paddle had a $1.2 \mathrm{~m}$ stroke length and was capable of generating most types of waves including periodic, solitary, leading depression- $\mathrm{N}$ waves, etc. The wave flume bed was made of marine plywood with sand paper having an equivalent Nikuradse roughness of $0.25 \mathrm{~mm}$ affixed over it. A shear plate apparatus used in previous studies (Barnes et al., 2009; Guard et al., 2010; Seelam et al., 2011) is used with the plate affixed with the same sand paper as that on the flume bed. The shear plate apparatus, fixed flush with the flume bed, houses a shear plate ( $0.1 \mathrm{~m}$ long; $0.25 \mathrm{~m}$ wide; $1.21 \mathrm{~mm}$ thick) supported on tubular sway legs, with a provision to measure displacement of the plate (resolution of $0.001 \mathrm{~mm}$ ) by a non-intrusive Indykon ${ }^{\circledR}$ eddy-current displacement sensor. The still water depth above the shear plate apparatus in the experiments ranged between 0.105 and $0.21 \mathrm{~m}$.
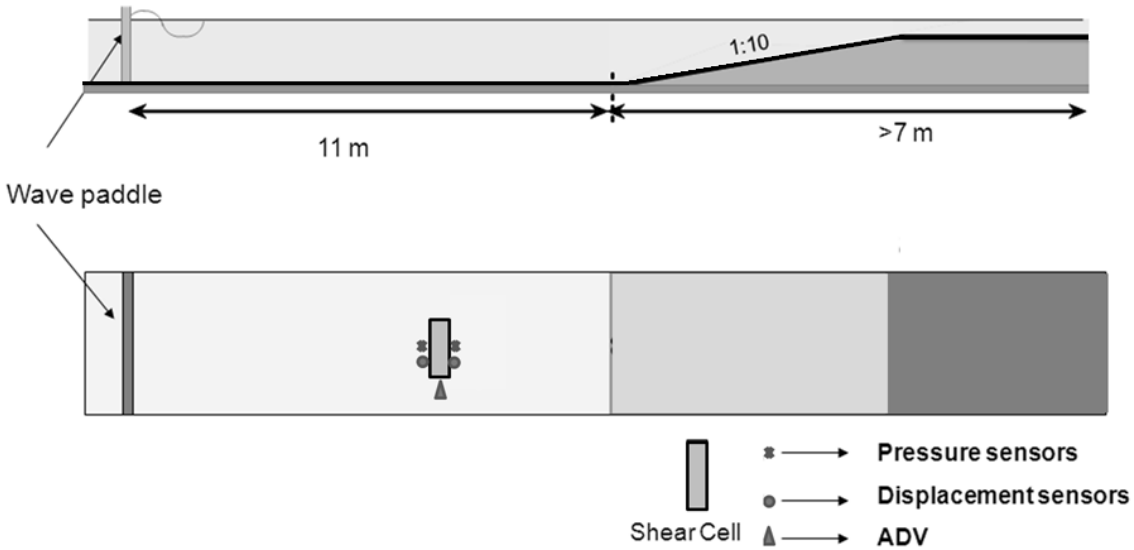

Figure 1. Experimental setup showing locations of shear plate apparatus (shear cell), pressure sensors, ADV, wave gauges (displacement sensors) and wave paddle; (figure not to scale; modified from Seelam et al., 2011).

Horizontal flow velocity under solitary waves was measured at $50 \mathrm{~Hz}$ sampling rate, $1 \mathrm{~cm}$ above the flume bed, using a SONTEK ${ }^{\circledR}$ 2D 16MHz Micro-Acoustic Doppler Velocimeter (ADV). The ADV has a sampling volume of $\sim 85.9 \mathrm{~mm}^{3}(6.2 \mathrm{~mm}$ high; $4.2 \mathrm{~mm} \mathrm{dia}$.) at an approximate horizontal distance of 5 $\mathrm{cm}$ from the sensors. The ADV is capable of measuring $1 \mathrm{~mm} / \mathrm{s}$ to $2.5 \mathrm{~m} / \mathrm{s}$ flow velocities with $1 \%$ accuracy for velocities $<1.7 \mathrm{~m} / \mathrm{s}$. The surface elevation was measured using Microsonic ${ }^{\circledR}$ acoustic displacement sensors (wave gauges) placed above the water surface. These are non-intrusive gauges capable of measuring water level displacements in a detection zone between $60-350 \mathrm{~mm}$, with an accuracy $<2 \%$ of measured values (Microsonic, 2005). The gauges were placed about $100 \mathrm{~mm}$ apart, evenly spaced upstream and downstream of the shear plate apparatus, coinciding with the edges of the shear plate. The data is synchronously collected from all the sensors using a National Instrumentation ${ }^{\circledR}$ data acquisition system. The wave generating program controls the starting and ending of the wave generation as well as the data collection.

Solitary waves were generated in the flume by providing appropriate voltage signals to the wave paddle through the wave generation software. Typical wave paddle displacements and their corresponding non-breaking waves generated are shown in Fig. 2; whereas, the wave paddle displacement and their corresponding breaking solitary bores are shown in Fig. 3. Similar waves generated in previous study of Seelam et al. (2011) were used. A total of 114 waves generated for various water depths are considered in this study, the range of the wave conditions are given in Tables 1 and 2 . 


\section{Bed shear stress}

The shear stress apparatus measures the horizontal displacement of the shear plate, which is due to the total force $\left(\tau_{T}\right)$ exerted by the solitary wave on the shear plate. The measured displacement is converted to total force on the plate using prior calibration coefficients of the shear plate. This total force comprises of both the pressure gradient force $\left(\tau_{p r}\right)$ and bed shear stress $(\tau)$ exerted on the shear plate thickness $\left(t_{p}\right)$. The pressure gradient force (Eq. 1) is derived near the plate edges using an estimated dynamic pressure derived from surface elevation $(\eta)$ for non-hydrostatic conditions (Nielsen, 2009), using an explicit approximation to linear dispersion relation (Fenton and McKee, 1990) in estimating the wave number. The bed shear stress is then obtained from deduction of $\tau_{p r}$ from $\tau_{T}$.

$$
\tau_{p r}=-\rho g \frac{\partial \eta}{\partial x} t_{p}
$$

The above method of estimating the pressure gradient force has been successfully used in earlier studies (Barnes et al., 2009; Grass et al., 1995; Ippen and Mitchell, 1957; Riedel, 1972; Seelam and Baldock, 2011; Seelam et al., 2011). For smooth bed experiments, the pressure gradient force derived from surface elevation was reduced by $65 \%$ to obtain a better comparison of bed shear stress with theoretical study of Liu et al. (2007). In this study similar reduction of the pressure gradient is employed.

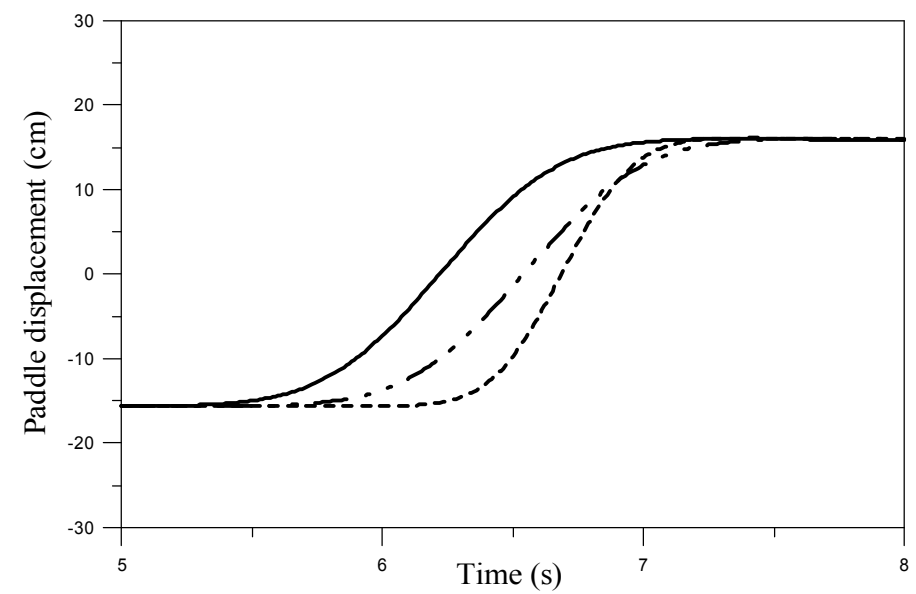

(a)

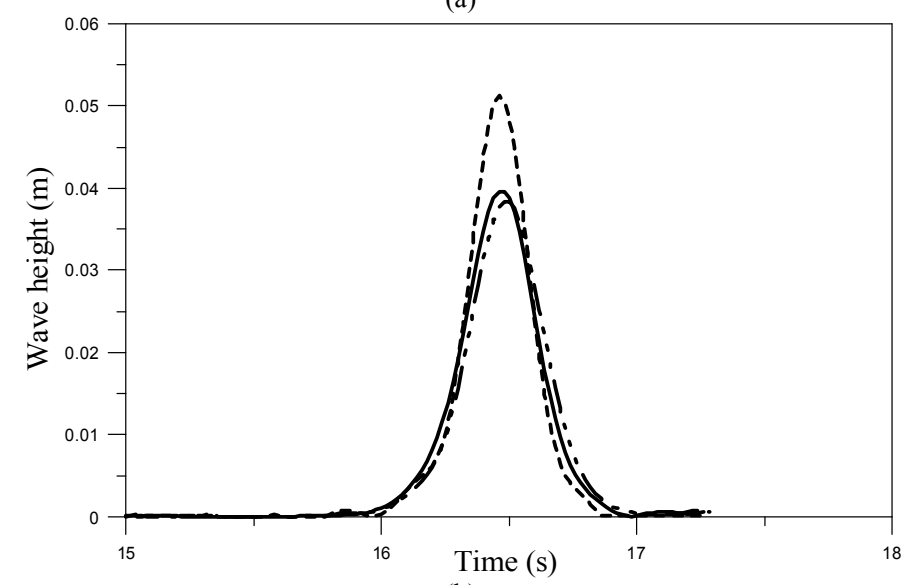

(b)

Figure 2. Paddle displacement and corresponding wave profile generated for non-breaking solitary waves. (a) wave paddle displacement (b) wave profile; and .... correspond to error wave function and corresponds to solitary wave function. 


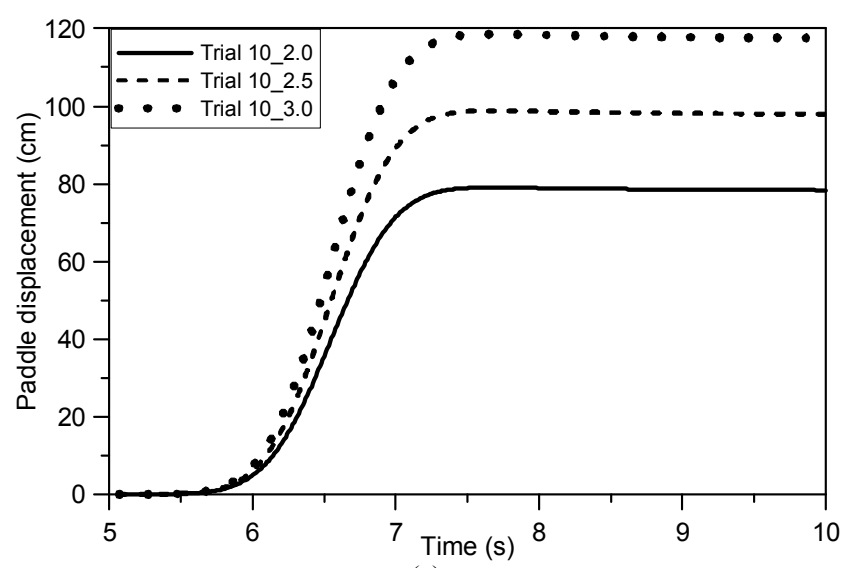

(a)

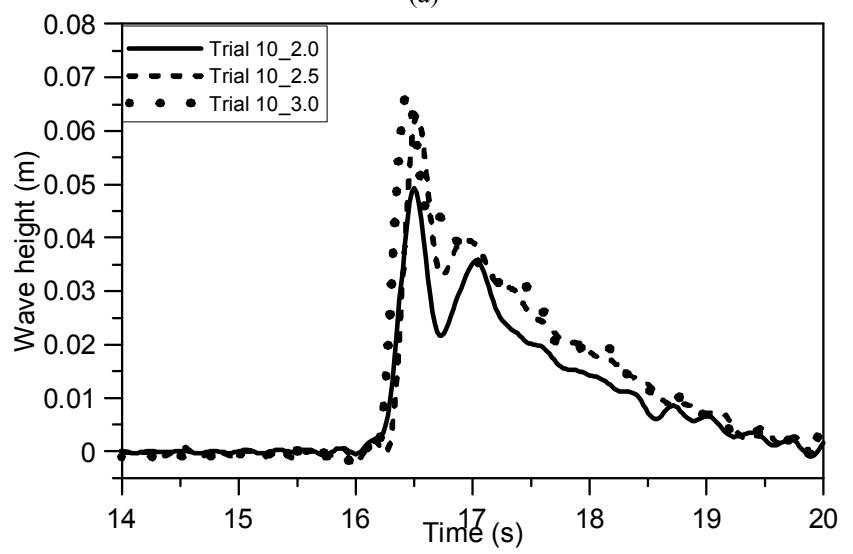

(b)

Figure 3. Wave paddle displacement and corresponding wave profile generated for breaking solitary bores (a) paddle displacement (b) wave profile.

Reynolds number $\left(R_{e}\right)$ for perfect solitary waves can be estimated using methods described in Ippen and Mitchell (1957) or Sumer et al. (2010). However, for solitary waves and bores that deviate from the theoretical waves, the Reynolds number can be estimated using the semi-excursion length derived by integrating the free stream velocity up to a cut off value, as estimated by Seelam et al. (2011).

\section{Analytical model}

Bed shear stress $(\tau)$ for laminar conditions is given by Newton's formula (e.g., Fredsøe and Deigaard, 1992; Nielsen, 1992) which is equal to the product of the local velocity gradient $\left(\frac{\partial u}{\partial z}\right)$, viscosity $(v)$ and density of the fluid $(\rho)$, as in Eq. (2). For steady and uniform flows the bed shear is proportional to the surface slope $(S)$ as in Eq. (3). For steady turbulent flows, analogous to laminar flow and considering the eddy viscosity concept, the relationship between the bed shear stress and velocity can be written as Eq. (4).

$$
\begin{gathered}
\tau=\rho v \frac{\partial u}{\partial z} \\
\tau=\rho g S(d-z) \\
\tau=\rho v_{t} \frac{\partial u}{\partial z}
\end{gathered}
$$

where, $\mathrm{g}$ is the acceleration due to gravity, $\mathrm{d}$ is the water depth, $\mathrm{z}$ is the elevation above the bed and $v_{t}$ is the eddy viscosity.

The bed shear stress was modeled using convolution integration of acceleration approach presented in Liu et al. (2007) and applying the numerical formulation of Torsvik and Liu (2007). Liu (2006) adapted the method of perturbation expansion of velocity field in the bottom boundary layer for long 
wave propagation and employed an eddy viscosity model assumed to be a power function of vertical elevation inside the boundary layer, as in Eq. (5).

$$
v_{t}=v\left[\frac{z}{z_{0}}\right]^{p}
$$

where, $\mathrm{z}_{0}$ is roughness height, $\mathrm{z}$ is depth, $v$ is kinematic viscosity and $p$ is the power with which $\left(z / z_{o}\right)$ varies. The leading order bed shear stress can be expressed as convolution integral of the depth integrated average horizontal velocity, $\bar{u}$, assuming the initial velocity to be zero.

$$
\tau=-\frac{q(1-q)^{2 q-1}}{\Gamma(q+1)} \int_{0}^{t} \frac{\partial \bar{u}(x, T) / \partial T}{(t-T)^{q}} d T
$$

where, $q$ and $p$ are related by $q=(1-p) /(2-p) ; x, T$ are the distance and time variables respectively; $t$ is the wave period and $\Gamma$ is the gamma function. For solitary waves, Liu et al. (2007) showed that the linearized boundary layer solutions are adequate to describe the bed shear stress in the boundary layer and the nonlinear effects are insignificant. The bed shear stress for long waves, assuming that the initial velocity is zero is given by convolution integration of local acceleration as in Eq. (7).

$$
\tau=\rho \sqrt{\frac{v_{t}}{\pi}} \int_{0}^{t} \frac{\partial u / \partial T}{(t-T)^{1 / 2}} d T
$$

The integrand in Eq. (7) is weighted by the function $(t-T)^{-\mathrm{q}}$ for $0<\mathrm{t}<\mathrm{T}$ and for $q=1 / 2 ; p=0$ which yields $v_{t}=v$ from Eq. (5). However, as indicated in the previous study (Seelam et al., 2011) the dependence of bed roughness and time scale of motion on the magnitude of $v_{t}$ is thus far untested in this method.

Three eddy viscosity formulations were used in determining the bed shear stress, as was considered for smooth bed in an earlier study (Seelam et al., 2011). The first formulation considered the eddy viscosity to be a constant equal to the kinematic viscosity of the fluid, which relates to laminar regime $(q=1 / 2$, or $p=0$, in Eq.5). This formulation is further referred in this paper as Conv-1 model. In the second formulation, referred as Conv-2 model, analogous to the steady flow condition, the eddy viscosity is considered to vary linearly with shear velocity $\left(u_{*}\right)$ and an elevation parameter $\left(\mathrm{z}_{1}\right)$ related to bed roughness $(r)$ and water particle excursion $(A)$ (Eq.8). The roughness of the sand paper considered in this experiment has an equivalent Nikuradse roughness of $0.25 \mathrm{~mm}$. The shear velocity, $u_{*}$, is estimated using Eq. (9) and the parameter $z_{1}$ is estimated using Eq. (10) as in Nielsen (1992), with $k_{l}$ being a constant with a value of 0.45 .

$$
\begin{gathered}
v_{t}=k_{1} u_{*} z_{1} \\
u_{*}=\sqrt{\frac{|\tau|}{\rho}} \\
z_{1}=0.09 \sqrt{r A}
\end{gathered}
$$

The convolution approach was modified as for turbulent flows, by taking $q=1 / 8$ (or $p=6 / 7$ ) and an eddy viscosity which is a function of the bed shear stress itself. This third method by taking $q=1 / 8$ and the eddy viscosity as given by Liu (2006) (Eq. 11) is referred in this paper as Conv-3 model.

$$
v_{t}=\frac{7 v}{8.7}\left(\frac{z_{1} u_{*}}{v}\right)^{6 / 7}
$$

Of the three models adopted for eddy viscosity to estimate the bed shear stress over a smooth bed (Seelam et al., 2011), the model with a constant viscosity performed better compared to other two eddy viscosity models as the flow regime for smooth bed experiments was mostly laminar. These models are now tested for rough bed experiments wherein the flow regimes are not necessarily laminar. As can be seen from Eq. $(7-11)$, the parameters required to measure the bed shear stresses are free stream velocity $(u)$ or shear velocity $\left(u_{*}\right)$, roughness $(r)$ and semi-excursion length $(A)$. The shear velocity can be obtained from the free stream velocity if log-law or law of the wall is assumed in the boundary layer. The physical bed roughness height can be measured whereas the semi-excursion length is estimated by integrating the velocity up to the peak, which has been explained in detail in our previous study (Seelam et al., 2011). 


\section{Friction factors}

Jonsson (1966) used wave friction factor $(f)$, free stream velocity $(u)$ and fluid density $(\rho)$ to estimate bed shear stress $(\tau$ ) using Eq. (12a), the quadratic drag law.

$$
\begin{gathered}
\tau=\frac{1}{2} \rho f u^{2} \\
f=\frac{2 \tau}{\rho u^{2}}
\end{gathered}
$$

This formulation of bed shear stress using squared free stream velocity is good for steady flows where the phase difference between the free stream velocity and the bed shear stress can be ignored. However, for unsteady flows, where the phase difference between $u$ and $\tau$ varies, the quadratic drag law therefore cannot be applied per se. The friction factors often derived from quadratic law (Eq. 12b), without considering the phase difference, produce values that do not corresponding to either maximum shear stress or maximum velocity, because the velocity corresponding to maximum shear stress need not be the maximum velocity and vice versa.

For laminar flow under a solitary wave, an average friction factor, $f_{w}$, derived from the definition of bed shear stress, applicable for the entire length of the wave, as evaluated by Suntoyo and Tanaka (2009) reads $f_{w}=1.56 / \sqrt{R_{e}}$. The friction factor for oscillatory waves over flat bed till a $R_{e}$ of $3 \times 10^{5}$ is well described by $f_{w}=2 / \sqrt{R_{e}}$ (Nielsen, 1992; Kamphuis, 1975). In this study, the friction factors were derived using Eq. 12b, with the velocity being either the peak velocity or the corresponding instantaneous velocity, and are plotted on a Stanton-type diagram of the oscillatory wave data for the maximum bed shear stress estimates.

\section{Phase difference}

It has been well established that the phase difference between the bed shear stress and free stream velocity is significant in estimation of sediment transport (see e.g., Nielsen, 2006; Nielsen and Guard, 2010). The phase difference between the peak velocity and the peak bed shear stress is considered in this study. In order to estimate this phase difference in degrees, the time elapsed between $2.5 \%$ of the peak velocity and the peak free stream velocity, in the forward direction, is considered as the effective half wave period. This half-wave period corresponds to $180^{\circ}$. The time difference between the peak bed shear stress and the peak velocity in seconds is converted to degrees, considering the equivalent wave period of the solitary wave. Applying this method, the phase differences between the peak bed shear stress and the peak velocity are estimated from the experiments as well as the analytical model with the eddy viscosity formulations.

\section{RESULTS AND DISCUSSION}

Measured and derived parameters for non-breaking solitary type waves and breaking solitary bores over a horizontal rough bed are presented in this paper. The non-breaking wave parameters wave height to water depth ratio $(\gamma)$, maximum measured free stream velocity $\left(u_{\max }\right)$, Reynolds number $\left(R_{e}\right)$ at maximum free stream velocity, maximum positive total shear stress $\left(\tau_{T_{-} \text {max }}\right)$, maximum negative total shear stress $\left(\tau_{T \_ \text {min }}\right)$ and semi-excursion length to water depth ratio $(A / d)$ are presented in Table.1. The breaking wave parameters are presented in Table. 2 except the $\tau_{T_{-} \min }$. The $\tau_{T_{-} \text {min }}$ is hereinafter referred as minimum total shear stress. In an earlier study (Seelam et al., 2011), the method used to estimate semi-excursion length and thus the Reynolds number was validated. A non-linear relationship between Froude number, $F_{r}$, and $\gamma$ is seen for the experimental data in this study (Fig.4), with the best fit following a power law $\left(F_{r}=0.55 \gamma^{0.75}\right)$. Even though the flow regime is non-linear, the maximum total shear stress $\left(\tau_{T_{-} \max }\right)$ measured using the shear plate is found to be linearly proportional to $\gamma$, where $\tau_{T_{\text {max }}}=5 \gamma$ (Fig.5), whereas it was $\tau_{T_{\text {max }}}=3.5 \gamma$ for smooth bed experiments. A timehistory of a typical non-breaking solitary wave surface elevation, corresponding free stream velocity and the total shear stress for both the smooth and rough beds are show in Fig. 6. 


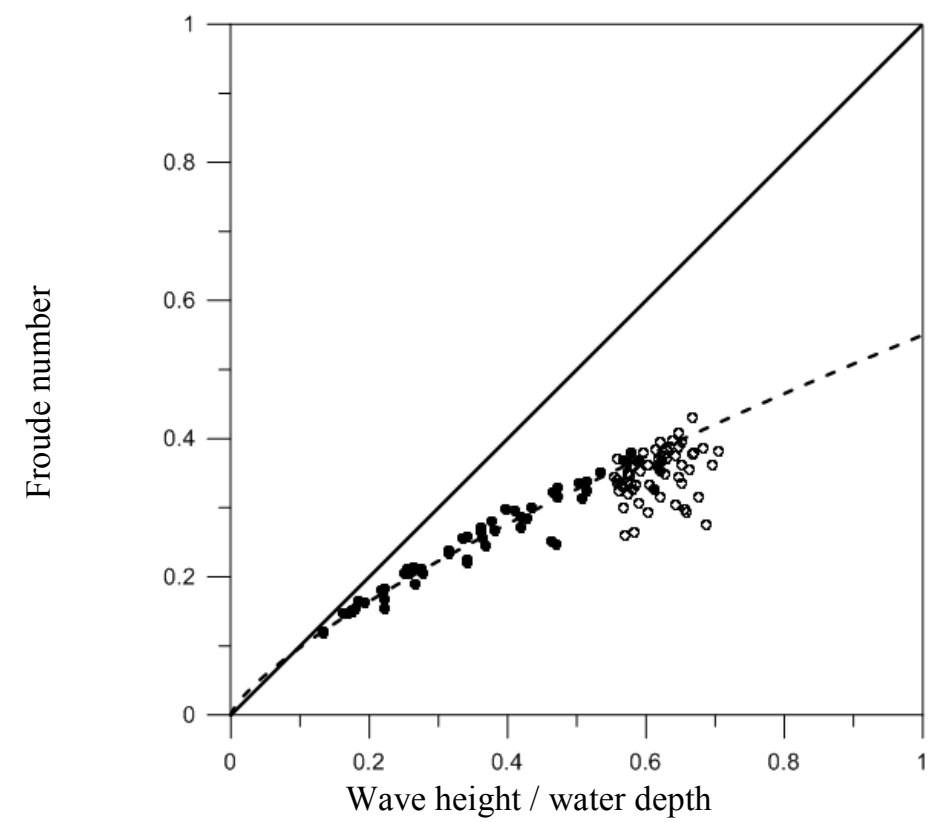

Figure 4. Relationship between Froude number, $F_{r}=u_{\max } / \sqrt{g d}$ and wave height to water depth ratio, $\boldsymbol{\gamma}$. Solid line is linear wave theory $\left(u_{\max }=\eta_{\max } \sqrt{g / d} ; F_{r}=\gamma\right) ;--.-$ - best fit $\left(F_{r}=0.55 \gamma^{0.75}\right)$; solid circles - non-breaking waves; hollow circles - breaking waves.

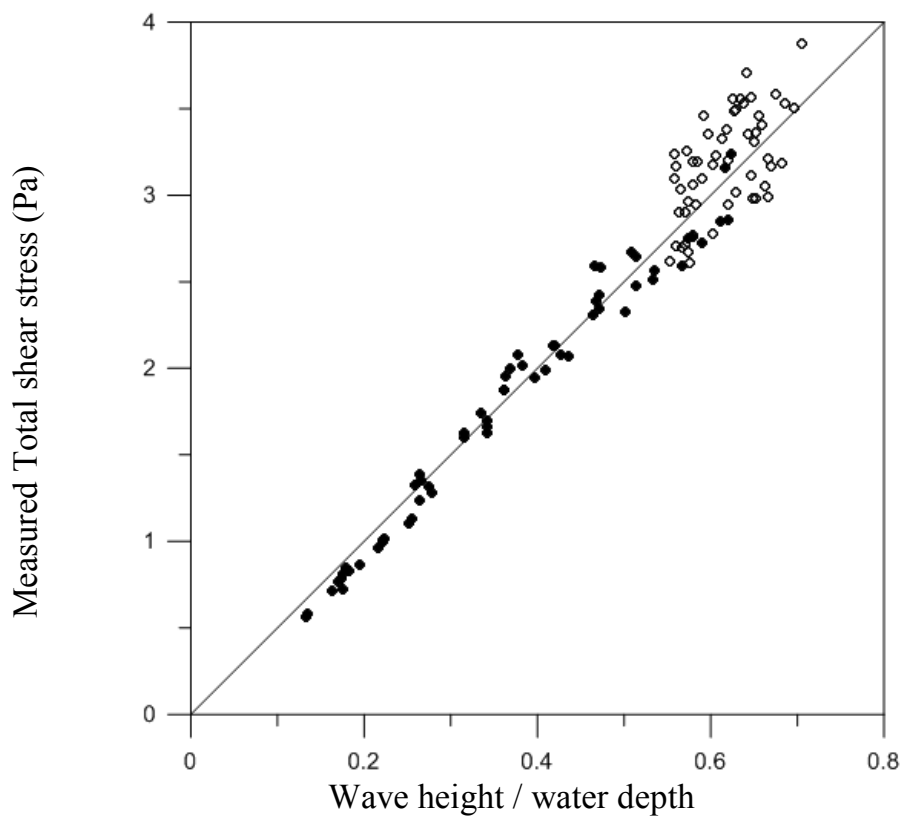

Figure 5. Relationship between maximum measured total shear stress, $\tau_{\mathrm{T}}$ and wave height to water depth ratio, $\gamma$. Solid line is line of best fit $\left(\tau_{T}=5 \gamma ; \mathbf{R}^{2}=0.99\right)$. Solid circles - non-breaking waves; hollow circles breaking waves.

The total shear stress leads the free stream velocity in the rough bed experiments carried out in this study (Fig. 6b), which is also observed in earlier studies (e.g., Ippen et al., 1955; Liu et al., 2007; Seelam and Baldock, 2009; Seelam and Baldock, 2011; Seelam et al., 2011; Sumer et al., 2008). Similar to the previous study for smooth bed experiments by the authors (Seelam et al., 2011), even though the free stream velocity does not go negative, the total shear stress changes its sign due to negative pressure gradient during the deceleration phase (Sumer et al., 2008). The peak negative total 
shear stress and the peak positive total shear stress were observed to follow a linear relationship with the peak negative total shear stress being about 0.46 times the peak positive total shear stress for nonbreaking waves. No such correlation was observed for the breaking solitary waves (Fig. 7).

The bed shear stress, $\tau$, is derived by deducting the pressure gradient force, $\tau_{p r}$, from the Total Shear stress, $\tau_{T}$. The bed shear stress results for smooth bed experiments were well calibrated with the analytical model and the results are presented in Seelam et al., (2011). The model results were well within $10 \%$ of the measurements, thereby indicating the effectiveness of the analytical model for the smooth bed results. The phase differences between the measured data and the model results were however unsatisfactory, which was attributed to the differences in estimating the pressure gradient forces which were estimated using a linear wave theory relationship.

The rough bed experimental results are analysed using the similar method adopted for the smooth bed results. A comparison between the measured and the predicted total shear stress (Fig. 8) shows that the results obtained from using the eddy viscosity formulation as in Eq. 8 provide better correlation compared to the other two eddy viscosity formulations. In case of smooth bed experimental results, the Conv-1 model (using kinematic viscosity along with a value of $q=0.5$ ) provided a better estimate of the bed shear stress. However, for the rough bed experimental results, the Conv-2 model (i.e, $q=1 / 2$ and the eddy viscosity given by Eq. 8) is observed to provide a better comparison with the measured data. A further investigation on an optimum value of $\mathrm{q}$ was carried out and the best correlation between the measured and model data was obtained for $q=1 / 2.4$ (Fig. 9). However, the Conv-3 model with $q=1 / 8$ as well as the laminar solution model (Conv-1) did not provide good comparisons with the measured data.

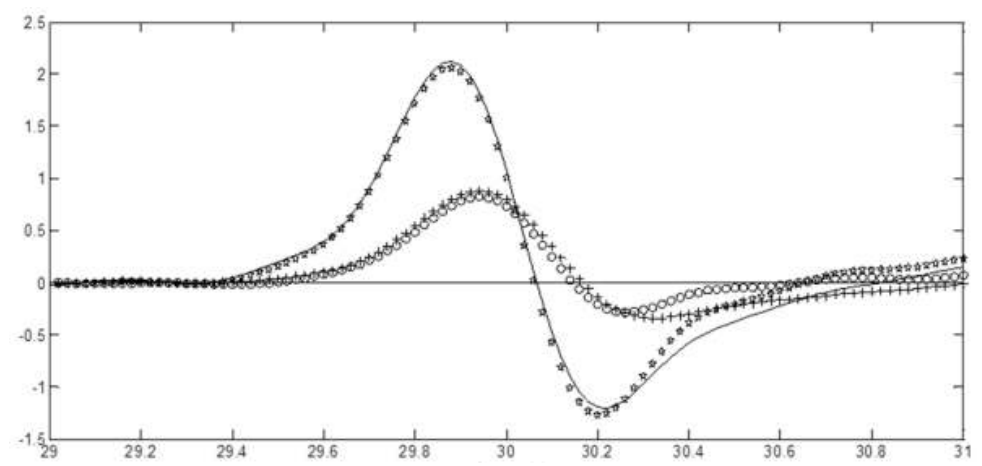

(a)

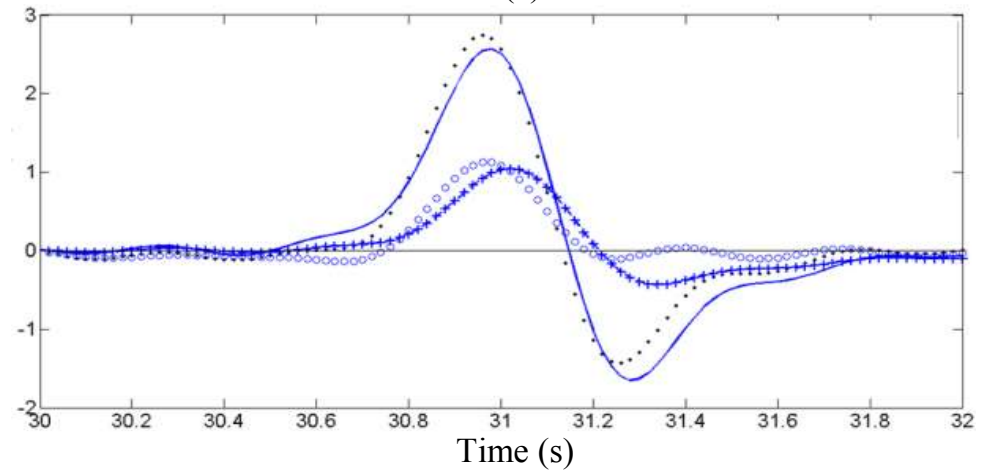

(b)

Figure 6. Time series of measured total shear stress (. . . .), modeled total shear stress ( _ $)$, modeled bed shear stress $(+++)$ and measured bed shear stress $(0 \circ 0)$ for solitary wave over $(a)$ smooth bed (modified from Seelam et al., 2011) and (b) rough bed. 


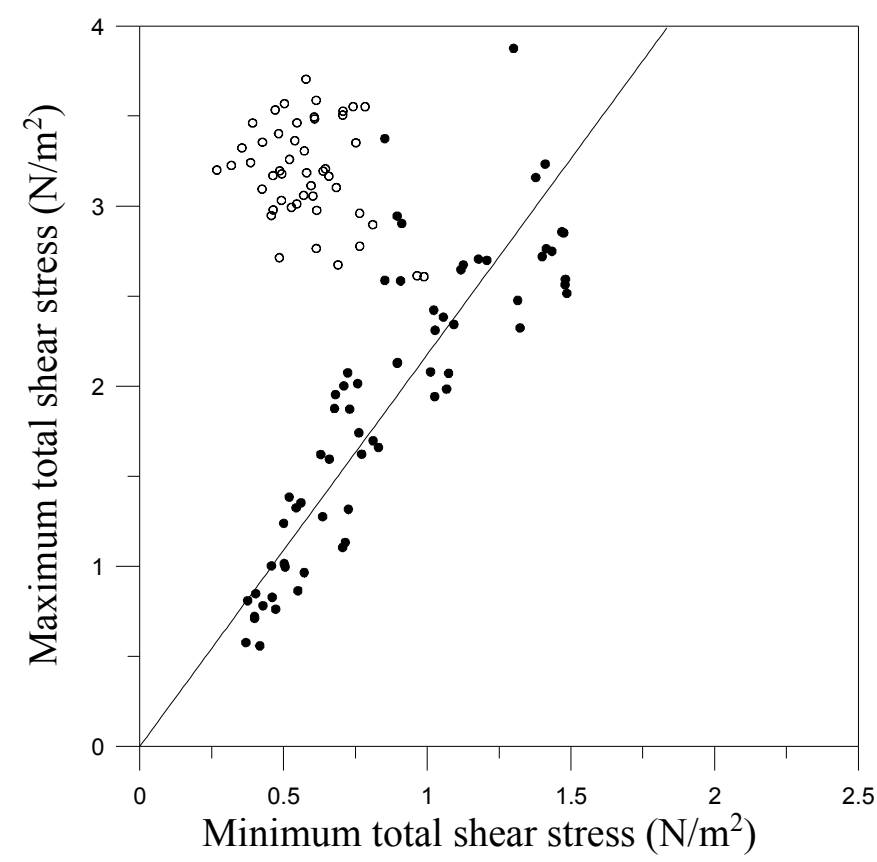

Figure 7. Comparison between measured maximum and minimum $\tau_{T}$, for horizontal rough bed. (best fit for non-breaking waves: $\tau_{\text {max }}=2.175 \tau_{T, \min } ; \mathbf{R}^{2}=0.96$ ). Solid circles - non-breaking waves; hollow circles breaking waves.

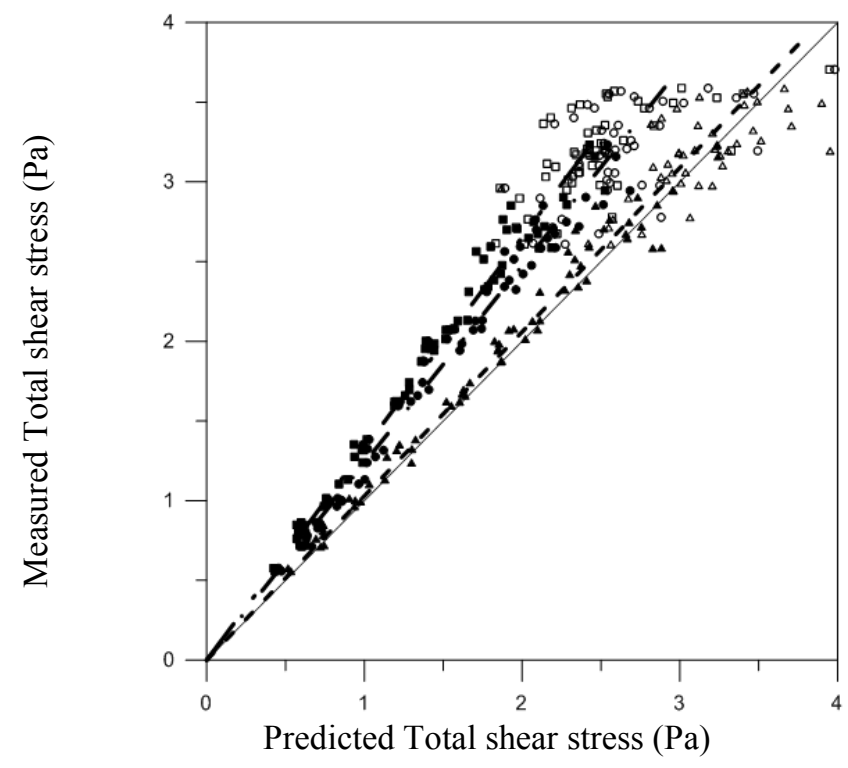

Figure 8. Comparison between measured and predicted total shear stress from convolution methods. Circles $q=1 / 2$ and $v_{e}=$ kinematic viscosity (laminar solution); Triangles $q=1 / 2.4$ and $v_{e}$ from Eq.8; Squares $q=1 / 8$ and $v_{e}$ from Eq.11. Solid symbols - non-breaking waves; Hollow symbols - breaking waves. 


\begin{tabular}{|c|c|c|c|c|c|c|c|}
\hline S.No & $\mathrm{H}_{\max } / \mathrm{d}$ & $\mathrm{U}_{\max }(\mathrm{m} / \mathrm{s})$ & $\mathrm{Re} @ \mathrm{U}_{\max }$ & $\tau_{T, \max }(\mathrm{Pa})$ & $\tau_{\mathrm{T}, \min }(\mathrm{Pa})$ & $\tau_{\max }(\mathrm{Pa})$ & $A / d$ \\
\hline 1. & 0.194 & 0.164 & 8500.0 & 0.864 & 0.550 & 0.431 & 0.495 \\
\hline 2. & 0.183 & 0.166 & 8217.0 & 0.828 & 0.461 & 0.407 & 0.474 \\
\hline 3. & 0.256 & 0.215 & 12502.6 & 1.133 & 0.715 & 0.791 & 0.555 \\
\hline 4. & 0.251 & 0.206 & 11389.1 & 1.105 & 0.705 & 0.475 & 0.526 \\
\hline 5. & 0.397 & 0.302 & 22092.7 & 1.942 & 1.026 & 0.852 & 0.696 \\
\hline 6. & 0.410 & 0.300 & 20513.6 & 1.984 & 1.067 & 0.892 & 0.649 \\
\hline 7. & 0.590 & 0.373 & 29874.3 & 2.720 & 1.400 & 1.052 & 0.762 \\
\hline 8. & 0.579 & 0.385 & 30019.6 & 2.764 & 1.414 & 1.394 & 0.743 \\
\hline 9. & 0.535 & 0.355 & 26665.1 & 2.563 & 1.480 & 1.311 & 0.716 \\
\hline 10. & 0.534 & 0.356 & 26945.4 & 2.515 & 1.486 & 1.208 & 0.720 \\
\hline 11. & 0.567 & 0.373 & 29194.7 & 2.594 & 1.481 & 1.291 & 0.746 \\
\hline 12. & 0.573 & 0.372 & 29302.4 & 2.749 & 1.434 & 1.122 & 0.748 \\
\hline 13. & 0.515 & 0.341 & 25616.7 & 2.476 & 1.315 & 1.004 & 0.715 \\
\hline 14. & 0.502 & 0.340 & 24855.1 & 2.324 & 1.323 & 0.971 & 0.696 \\
\hline 15. & 0.278 & 0.207 & 11162.8 & 1.276 & 0.636 & 0.541 & 0.515 \\
\hline 16. & 0.275 & 0.214 & 12161.2 & 1.316 & 0.726 & 0.550 & 0.542 \\
\hline 17. & 0.428 & 0.289 & 19324.4 & 2.079 & 1.012 & 0.832 & 0.634 \\
\hline 18. & 0.435 & 0.303 & 21331.3 & 2.071 & 1.075 & 0.886 & 0.663 \\
\hline 19. & 0.612 & 0.331 & 21792.9 & 2.851 & 1.475 & 1.319 & 0.627 \\
\hline 20. & 0.621 & 0.357 & 26639.0 & 2.857 & 1.468 & 0.993 & 0.710 \\
\hline 21. & 0.163 & 0.182 & 13103.2 & 0.711 & 0.399 & 0.361 & 0.465 \\
\hline 22. & 0.170 & 0.182 & 13101.5 & 0.762 & 0.473 & 0.395 & 0.465 \\
\hline 23. & 0.217 & 0.222 & 16780.5 & 0.965 & 0.572 & 0.515 & 0.486 \\
\hline 24. & 0.222 & 0.225 & 17916.9 & 0.996 & 0.506 & 0.523 & 0.518 \\
\hline 25. & 0.336 & 0.315 & 29548.4 & 1.741 & 0.762 & 0.909 & 0.604 \\
\hline 26. & 0.342 & 0.318 & 31568.9 & 1.660 & 0.831 & 0.858 & 0.642 \\
\hline 27. & 0.468 & 0.397 & 44083.5 & 2.383 & 1.056 & 1.128 & 0.715 \\
\hline 28. & 0.472 & 0.388 & 40575.9 & 2.343 & 1.093 & 1.150 & 0.676 \\
\hline 29. & 0.617 & 0.443 & 47263.6 & 3.159 & 1.378 & 1.397 & 0.688 \\
\hline 30. & 0.624 & 0.453 & 49027.2 & 3.233 & 1.411 & 1.521 & 0.701 \\
\hline 31. & 0.419 & 0.352 & 33863.2 & 2.128 & 0.896 & 1.023 & 0.620 \\
\hline 32. & 0.420 & 0.332 & 31986.7 & 2.132 & 0.897 & 0.999 & 0.621 \\
\hline 33. & 0.514 & 0.400 & 40975.6 & 2.647 & 1.118 & 1.186 & 0.661 \\
\hline 34. & 0.508 & 0.387 & 38532.9 & 2.674 & 1.125 & 1.125 & 0.643 \\
\hline 35. & 0.223 & 0.190 & 13106.8 & 1.015 & 0.502 & 0.469 & 0.447 \\
\hline 36. & 0.222 & 0.206 & 14604.3 & 1.003 & 0.458 & 0.499 & 0.454 \\
\hline 37. & 0.343 & 0.278 & 24208.7 & 1.622 & 0.772 & 0.815 & 0.562 \\
\hline 38. & 0.342 & 0.272 & 20693.9 & 1.697 & 0.812 & 0.797 & 0.492 \\
\hline 39. & 0.464 & 0.308 & 24560.3 & 2.311 & 1.027 & 1.171 & 0.513 \\
\hline 40. & 0.471 & 0.304 & 22098.3 & 2.422 & 1.023 & 1.024 & 0.469 \\
\hline 41. & 0.133 & 0.168 & 12354.2 & 0.558 & 0.418 & 0.406 & 0.347 \\
\hline 42. & 0.134 & 0.172 & 13386.4 & 0.576 & 0.369 & 0.389 & 0.374 \\
\hline 43. & 0.176 & 0.214 & 19441.0 & 0.721 & 0.399 & 0.483 & 0.434 \\
\hline 44. & 0.175 & 0.216 & 19586.7 & 0.809 & 0.376 & 0.501 & 0.436 \\
\hline 45. & 0.264 & 0.307 & 34813.7 & 1.239 & 0.501 & 0.766 & 0.540 \\
\hline 46. & 0.259 & 0.293 & 31582.7 & 1.325 & 0.544 & 0.854 & 0.513 \\
\hline 47. & 0.361 & 0.389 & 49191.3 & 1.876 & 0.677 & 1.144 & 0.604 \\
\hline 48. & 0.361 & 0.379 & 46547.9 & 1.873 & 0.730 & 1.149 & 0.586 \\
\hline 49. & 0.466 & 0.462 & 63475.4 & 2.587 & 0.853 & 1.398 & 0.654 \\
\hline 50. & 0.473 & 0.471 & 64614.8 & 2.585 & 0.908 & 1.320 & 0.655 \\
\hline 51. & 0.316 & 0.341 & 39635.3 & 1.621 & 0.630 & 0.978 & 0.557 \\
\hline 52. & 0.316 & 0.334 & 38700.1 & 1.595 & 0.660 & 0.936 & 0.555 \\
\hline 53. & 0.382 & 0.383 & 48640.4 & 2.015 & 0.758 & 1.175 & 0.604 \\
\hline 54. & 0.377 & 0.401 & 49368.3 & 2.077 & 0.724 & 1.204 & 0.584 \\
\hline 55. & 0.179 & 0.220 & 19368.6 & 0.848 & 0.403 & 0.497 & 0.420 \\
\hline 56. & 0.174 & 0.215 & 19582.4 & 0.781 & 0.429 & 0.448 & 0.435 \\
\hline 57. & 0.264 & 0.302 & 35829.8 & 1.384 & 0.520 & 0.874 & 0.565 \\
\hline 58. & 0.266 & 0.270 & 23390.8 & 1.353 & 0.561 & 0.860 & 0.416 \\
\hline 59. & 0.368 & 0.350 & 39806.0 & 2.002 & 0.710 & 1.215 & 0.541 \\
\hline 60. & 0.364 & 0.365 & 43222.6 & 1.953 & 0.681 & 1.170 & 0.567 \\
\hline
\end{tabular}




\begin{tabular}{|c|c|c|c|c|c|c|}
\hline S.No & $\mathrm{H}_{\max } / \mathrm{d}$ & $\mathrm{U}_{\max }(\mathrm{m} / \mathrm{s})$ & $\operatorname{Re} @ U_{\max }$ & $\tau_{T, \max }(\mathrm{Pa})$ & $\tau_{T, \min }(\mathrm{Pa})$ & $\tau_{\max }(\mathrm{Pa})$ \\
\hline 1. & 0.554 & 0.349 & 23896 & 2.614 & 1.178 & 0.652 \\
\hline 2. & 0.576 & 0.351 & 24593 & 2.608 & 0.980 & 0.666 \\
\hline 3. & 0.602 & 0.368 & 26271 & 2.777 & 0.582 & 0.678 \\
\hline 4. & 0.575 & 0.354 & 24404 & 2.674 & 0.897 & 0.654 \\
\hline 5. & 0.620 & 0.399 & 33219 & 2.948 & 1.123 & 0.789 \\
\hline 6. & 0.648 & 0.393 & 30898 & 2.979 & 0.944 & 0.747 \\
\hline 7. & 0.647 & 0.414 & 38449 & 3.114 & 1.494 & 0.884 \\
\hline 8. & 0.667 & 0.437 & 40819 & 3.207 & 1.364 & 0.890 \\
\hline 9. & 0.567 & 0.304 & 20345 & 2.699 & 1.135 & 0.636 \\
\hline 10. & 0.560 & 0.343 & 24908 & 2.706 & 1.106 & 0.690 \\
\hline 11. & 0.579 & 0.340 & 24132 & 2.765 & 1.120 & 0.675 \\
\hline 12. & 0.571 & 0.342 & 22906 & 2.714 & 1.189 & 0.637 \\
\hline 13. & 0.652 & 0.400 & 34222 & 2.977 & 0.839 & 0.815 \\
\hline 14. & 0.629 & 0.376 & 28134 & 3.012 & 1.160 & 0.711 \\
\hline 15. & 0.669 & 0.386 & 33834 & 3.169 & 1.303 & 0.837 \\
\hline 16. & 0.666 & 0.381 & 30507 & 2.993 & 1.188 & 0.763 \\
\hline 17. & 0.663 & 0.360 & 28427 & 3.056 & 1.148 & 0.755 \\
\hline 18. & 0.683 & 0.392 & 35800 & 3.185 & 1.339 & 0.866 \\
\hline 19. & 0.575 & 0.394 & 35812 & 2.960 & 1.119 & 0.586 \\
\hline 20. & 0.564 & 0.408 & 40229 & 2.897 & 1.530 & 0.639 \\
\hline 21. & 0.565 & 0.409 & 37521 & 3.032 & 1.493 & 0.590 \\
\hline 22. & 0.580 & 0.402 & 38279 & 3.060 & 1.243 & 0.613 \\
\hline 23. & 0.620 & 0.388 & 37073 & 3.200 & 1.346 & 0.616 \\
\hline 24. & 0.605 & 0.409 & 39789 & 3.226 & 1.255 & 0.624 \\
\hline 25. & 0.651 & 0.446 & 47349 & 3.307 & 1.592 & 0.680 \\
\hline 26. & 0.696 & 0.446 & 50513 & 3.505 & 1.391 & 0.733 \\
\hline 27. & 0.584 & 0.325 & 26819 & 2.945 & 0.813 & 0.537 \\
\hline 28. & 0.570 & 0.320 & 26033 & 2.904 & 1.108 & 0.525 \\
\hline 29. & 0.591 & 0.377 & 32774 & 3.094 & 1.389 & 0.560 \\
\hline 30. & 0.603 & 0.361 & 32943 & 3.179 & 1.293 & 0.586 \\
\hline 31. & 0.659 & 0.360 & 28913 & 3.402 & 1.828 & 0.520 \\
\hline 32. & 0.643 & 0.375 & 47927 & 3.355 & 1.438 & 0.827 \\
\hline 33. & 0.652 & 0.413 & 50221 & 3.363 & 1.901 & 0.787 \\
\hline 34. & 0.687 & 0.339 & 26068 & 3.534 & 1.541 & 0.497 \\
\hline 35. & 0.655 & 0.367 & 31239 & 3.463 & 1.718 & 0.550 \\
\hline 36. & 0.676 & 0.389 & 35433 & 3.587 & 1.158 & 0.591 \\
\hline 37. & 0.560 & 0.465 & 55907 & 3.166 & 1.628 & 0.571 \\
\hline 38. & 0.559 & 0.481 & 62997 & 3.094 & 1.431 & 0.626 \\
\hline 39. & 0.585 & 0.478 & 60003 & 3.196 & 1.529 & 0.598 \\
\hline 40. & 0.573 & 0.504 & 66430 & 3.259 & 1.440 & 0.628 \\
\hline 41. & 0.591 & 0.507 & 73191 & 3.462 & 1.786 & 0.688 \\
\hline 42. & 0.628 & 0.501 & 59835 & 3.484 & 2.103 & 0.568 \\
\hline 43. & 0.648 & 0.493 & 64626 & 3.569 & 2.038 & 0.622 \\
\hline 44. & 0.642 & 0.538 & 82826 & 3.705 & 1.736 & 0.733 \\
\hline 45. & 0.704 & 0.546 & 74247 & 3.875 & 2.249 & 0.648 \\
\hline 46. & 0.619 & 0.533 & 75433 & 3.375 & 1.691 & 0.675 \\
\hline 47. & 0.580 & 0.515 & 75828 & 3.194 & 0.605 & 0.702 \\
\hline 48. & 0.559 & 0.532 & 79192 & 3.241 & 1.550 & 0.710 \\
\hline 49. & 0.597 & 0.545 & 85744 & 3.352 & 1.495 & 0.749 \\
\hline 50. & 0.613 & 0.551 & 82201 & 3.323 & 1.823 & 0.713 \\
\hline 51. & 0.629 & 0.549 & 85981 & 3.495 & 1.414 & 0.747 \\
\hline 52. & 0.637 & 0.569 & 87145 & 3.527 & 1.086 & 0.730 \\
\hline 53. & 0.634 & 0.556 & 89565 & 3.553 & 1.410 & 0.767 \\
\hline 54. & 0.626 & 0.537 & 79862 & 3.552 & 1.762 & 0.710 \\
\hline
\end{tabular}




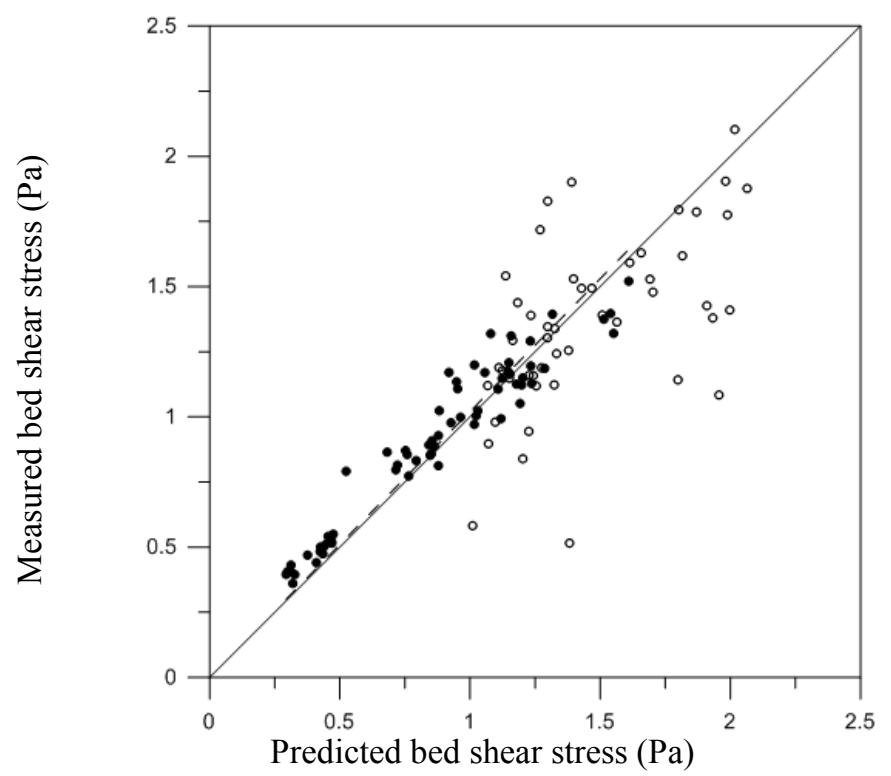

Figure 9. Comparison between measured and predicted bed shear stress from convolution method. Circles $q$ $=1 / 2.4$ in equation 5 and $v_{e}$ from Eq. 8; best fit line; $\left(\tau_{m}=1.015 \tau_{p} ; \mathbf{R}^{2}=\mathbf{0 . 9 8 8}\right)$. Solid symbols - nonbreaking waves; Hollow symbols - breaking waves.

The friction factors derived using Eq. $12 \mathrm{~b}$ at the maximum bed shear stress using maximum velocities varied between $6 / \sqrt{R_{e}}$ and $8 / \sqrt{R_{e}}$ and the friction factors derived using their corresponding instantaneous velocities varied between $8 / \sqrt{R_{e}}$ and $18 / \sqrt{R_{e}}$ (Fig. 10). The friction factors estimated in this experiment are in the flow regime representing laminar to transition region. It can be clearly seen that the breaking waves have higher velocities and hence higher Re. It is also seen that for both breaking and non-breaking waves the friction factors obtained using instantaneous velocity are comparatively larger than those obtained using the maximum velocity.

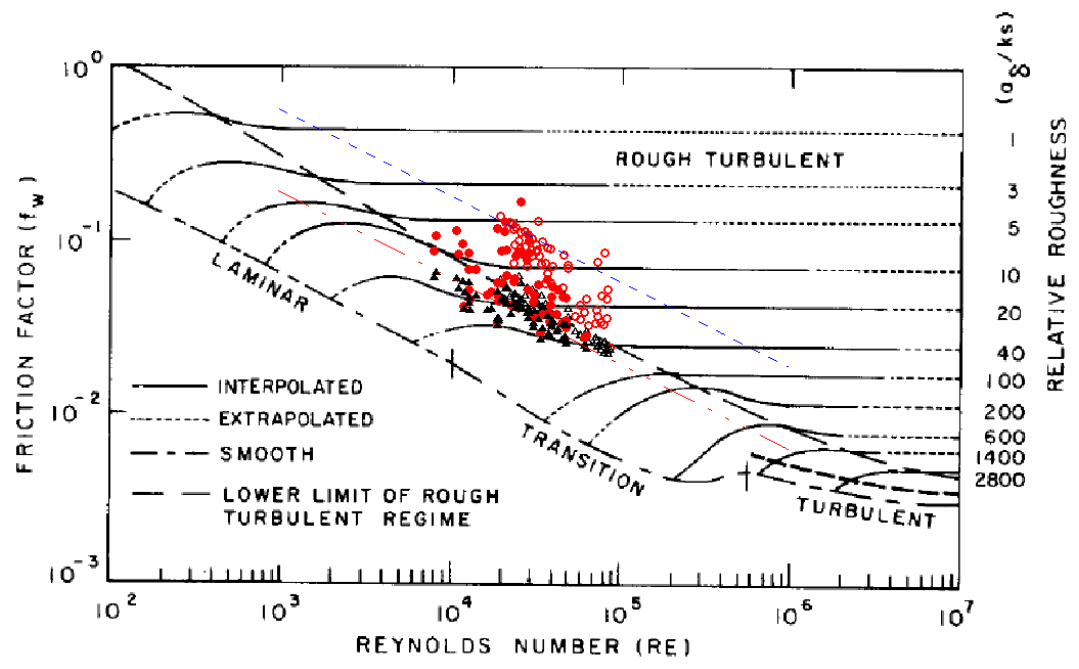

Figure 10. Wave friction factors at maximum bed shear stress plotted on stanton-type diagram of Kamphuis (1978). Circles (red color) represent the friction factors derived using instantaneous velocity and triangles

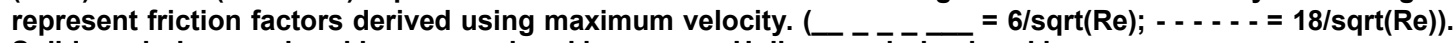
Solid symbols - non-breaking waves; breaking waves; Hollow symbols - breaking waves. 
The upper bound for the friction factors described by $f=18 / \sqrt{R_{e}}$ and the lower bound of $6 / \sqrt{R_{e}}$ are much higher than the average $f_{w}$ as given by Suntoyo and Tanaka (2009) which is $1.56 / \sqrt{R_{e}}$ as well as the values for smooth laminar oscillatory flow which is $2 / \sqrt{R_{e}}$. These results also suggest that the friction factors for horizontal rough bed could be estimated by simplified functions of $R_{e}$. However, the relationship between the roughness and the Reynolds number should be considered to come up with a proper model of friction factor under solitary waves on a rough bed.

The phase difference between the measured maximum bed shear stress and the maximum velocity and the phase difference obtained from Conv- 2 analytical model bed shear stress and the measured maximum velocity are compared to establish the applicability of the analytical model for solitary waves on a rough bed. The phase difference between maximum velocity and Conv-2 modelled bed shear stress for non breaking waves was around $30^{\circ}$ and for breaking waves it was about $48^{\circ}$. The prediction from the analytical model (Conv-2 model) for this phase difference is under-estimated by about $27 \%$ (Fig. 11).

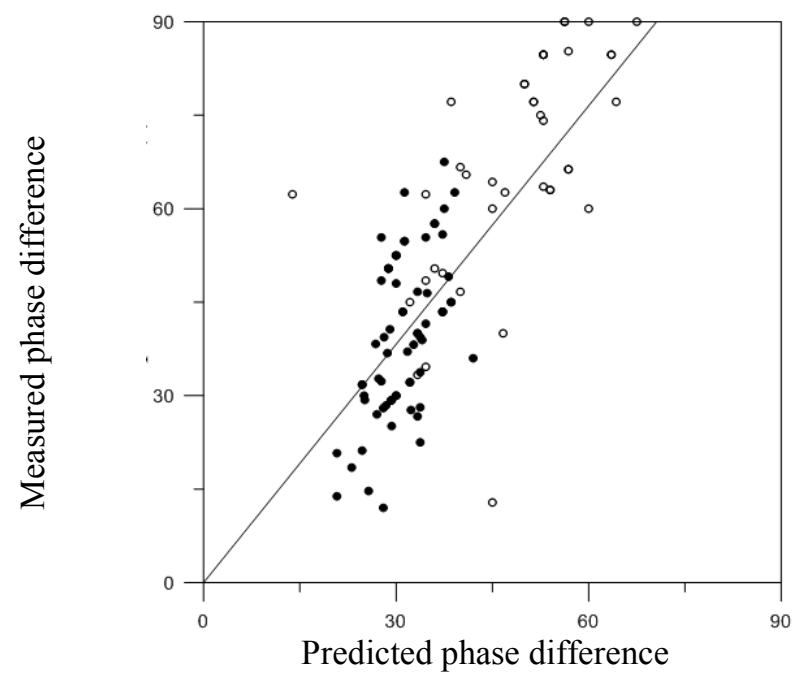

Figure 11. Plot of phase differences between measured $u_{\max }$ and measured $\tau_{\max }$ on $y$-axis and between measured $u_{\max }$ and predicted $\tau_{\max }$ using Conv-2 model (_ best fit line $y=1.275 x$ ); Solid symbols: nonbreaking waves; hollow symbols - breaking waves.

\section{CONCLUSIONS}

Laboratory investigations are carried out in a wave flume on solitary wave induced bed shear stresses on a rough bed using a shear plate apparatus. Both non-breaking and breaking type waves were studied, which showed that the breaking type waves were more energetic than the non-breaking waves. The Reynolds numbers ranged between 7600 and 60200; the Reynolds numbers pertaining to breaking waves being higher than non-breaking waves. A linear relationship existed between the relative wave height (wave height to water depth ratio) and the total shear stress both for breaking and non-breaking waves. The variation of Froude number with relative wave height was non-linear even though the total shear stress displayed linear relationship. A change in the sign of the total shear stress due to the adverse pressure gradients during the deceleration phase of the wave is clearly seen, similar to the earlier studied reported in the literature. The bed shear stress derived from the total shear stress did not show predominant negative shear stress during the deceleration phase compared to the smooth bed results.

Analytical model for solitary wave induced bed shear stress using free stream velocity and different eddy viscosity models including a model incorporating the bed roughness was tested on the experimental results. These tests showed that the analytical model based on convolution integration of the flow acceleration along with appropriate bed roughness model incorporated into the eddy viscosity terms provides good comparisons with the measured data. The model not only predicts the bed shear stress satisfactorily but also provides good estimates of the total shear stress when non-hydrostatic pressure gradient force terms are added. The friction factors were higher for both non-breaking as well as breaking waves when compared to the friction factors obtained from laminar oscillatory wave data. 
The analytical model derived phase difference between the maxima of bed shear stress and the velocity was found to be underestimated by about $27 \%$.

\section{ACKNOWLEDGMENTS}

The work carried out in this paper is part of a research project supported by CSIRO-Australia's Flagship Cluster Grant under Wealth from Oceans - Pipeline Hazards program. First author acknowledges the support of his parent institute, CSIR-National Institute of Oceanography, Goa, India and the financial support of Endeavour IPRS and UQRS during his stay at the UQ. This is NIO contribution No. 5247.

\section{REFERENCES}

Barnes, M.P., T. O'Donoghue, J.M. Alsina and T.E. Baldock. 2009. Direct bed shear stress measurements in bore-driven swash. Coastal Engineering, 56: 853-867.

Fenton, J.D. and W.D. McKee. 1990. On calculating the lengths of water waves. Coastal Engineering, 14: 499 - 513.

Fredsøe, J. and R. Deigaard. 1992. Mechanics of coastal sediment transport. Advanced series on ocean engineering - volume 3. World Scientific, $369 \mathrm{pp}$.

Grass, A.J., R.R. Simons, R.D. Maciver, M. Mansour-Tehrani and A. Kalopedis. 1995. Shear cell for direct measurement of fluctuating bed shear stress vector in combined wave/current flow. In: P.o.X.I. Congress (Editor), Hydraulic Research and its Applications next Century - HYDRA 2000, pp. 415-420.

Guard, P.A., P. Nielsen and T.E. Baldock. 2010. Bed shear stress in unsteady flow, 32nd International Conference on Coastal Engineering, Shangai, China, pp. 1-8.

Ippen, A.T., G. Kulin and M.A. Raza. 1955. Damping characteristics of the solitary wave. 16, Massachusetts Institute of Technology, Hydrodynamics Laboratory.

Ippen, A.T. and M.M. Mitchell. 1957. The damping of the solitary wave from boundary shear measurements. 23, Massachusetts Institute of Technology, Hydrodynamics Laboratory.

Jonsson, I.G. 1966. Wave boundary layer and friction factors, Proc. 10th Coastal Engineering Conference, Tokyo, Japan, pp. 127-148.

Keulegan, G.H. 1948. Gradual damping of solitary waves. U.S. Department of Commerce, National Bureau of Standards, Res. Pap. RP 1895 40: 487-498.

Liu, P.L.F. 2006. Turbulent boundary-layer effects on transient wave propagation in shallow water. Proceedings of the Royal Society London A, 462(2075): 3481-3491.

Liu, P.L.F., Y.S. Park and E.A. Cowen. 2007. Boundary layer flow and bed shear stress under a solitary wave. Journal of Fluid Mechanics, 574: 449-463.

Microsonic. 2005. Instruction manual. In: M. GmbH (Editor), Dortmund, Germany.

Naheer, E. 1978. The damping of solitary waves. Journal of Hydraulic Research, 16(3): 235-248.

Nielsen, P. 1992. Coastal bottom boundary layers. World Scientific, Singapore, 324 pp.

Nielsen, P. 2006. Sheet flow sediment transport under waves with acceleration skewness and boundary layer streaming. Coastal Engineering, 53(9): 749-758.

Nielsen, P. 2009. Coastal and estuarine processes. Advanced series on ocean engineering. World Scientific, New York, NJ (USA), 343 pp.

Nielsen, P. and P.A. Guard. 2010. Vertical scales and shear stresses in wave boundary layers over movable beds. 32nd International Conference on Coastal Engineering, Shangai, China, p.^pp. $1-8$.

Riedel, H.P. 1972. Direct mesasurement of bed shear stress under waves. Ph.D Thesis Thesis, Queens University, Kingston.

Seelam, J.K. and T.E. Baldock. 2009. Direct bed shear measurements under tsunami waves and breaking tsunami wavefronts, International Conference on Coastal Dynamics 2009, Tokyo, Japan.

Seelam, J.K. and T.E. Baldock. 2011. Comparison of bed shear under non-breaking and breaking solitary waves. The International Journal of Ocean and Climate Systems, 2(4): 259-278.

Seelam, J.K., P.A. Guard and T.E. Baldock. 2011. Measurement and modeling of bed shear stress under solitary waves. Coastal Engineering, 58(9): 937-947.

Shimozono, T., A. Okayasu and T. Mishima. 2010. On the bottom shear stress during long wave runup and backwash, International Conference on Coastal Engineering, pp. 1-14. 
Sumer, B.M., P.M. Jensen, L.B. Soerensen, J. Fredsøe, Liu, P. L. F. and S. Carstensen. 2010. Coherent structures in wave boundary layers. Part 2. Solitary motion. Journal of Fluid Mechanics, 646: 207-231.

Sumer, B.M., P.M. Jensen, L.B. Sorensen, J. Fredsøe and P.L.F. Liu. 2008. Turbulent solitary wave boundary layer. In: T.I.S.o.O.a.P.E. (ISOPE) (Editor), Eighteenth (2008) International Offshore and Polar Engineering Conference, Vancouver, BC, Canada, pp. 775-781.

Suntoyo and H. Tanaka. 2009. Numerical modeling of boundary layer flows for a solitary wave. Journal of Hydro-environment Research, 3: 129-137. 